\title{
Actores, redes y alimentos: Ia participación de la agricultura familiar y campesina en la alimentación escolar del Brasil y Colombia
}

\author{
Patricia Elena Giraldo Calderón \\ Universidade Federal do Rio Grande do Sul - Porto Alegre - Rio Grande do Sul - \\ Brasil \\ Catia Grisa \\ Universidade Federal do Rio Grande do Sul - Porto Alegre - Rio Grande do Sul - \\ Brasil
}

\section{Resumen}

Este artículo tiene por propósito comparar las redes de compras públicas para la agricultura campesina y familiar en los programas de alimentación escolar de los municipios de Granada (Antioquia-Colombia) y São Lourenço do Sul (Rio Grande do Sul-Brasil) en los años de 2016 y 2017. Para tal fin, se construyó un abordaje teórico-metodológico desde la perspectiva de las redes de política pública, articulado a dos metodologías, el Análisis de Redes Sociales y la comparación de Sistemas de Máxima Diferencia. Entre los resultados obtenidos se evidenció cómo los municipios, de contextos políticoinstitucionales diferenciados, convergen en relaciones y actores semejantes para garantizar compras públicas en la alimentación escolar. No obstante esta convergencia, también fue posible observar diferencias en cuanto al papel del Estado y sus políticas, más fuerte y de mayor trayectoria en Brasil que en Colombia; la cooperación internacional, más fuerte e importante en Colombia que en Brasil; el papel de los actores sociales, fuertes y organizados en ambos municipios pero con mayor soporte institucional en Brasil que en Colombia; y, finalmente, un liderazgo de diferentes actores y agentes locales para implementar las compras públicas en la alimentación escolar que incluyan a los agricultores campesinos y familiares.

Palabras clave: Alimentación Escolar. Redes de Política Pública. Política Comparada. Brasil. Colombia. 


\title{
Actors, networks and food: participation of family farming and peasant in school meal programs in Brazil and Colombia
}

\begin{abstract}
The objective of this paper is to compare the networks of public purchase for peasant and family farming in school meal programs in the municipalities of Granada (AntioquiaColombia) and São Lourenço do Sul (Rio Grande do Sul-Brazil) in 2016 and 2017. A theoretical approach was built from the perspective of policy networks and articulated two methodologies, the Analysis of Social Networks and the comparison of Maximum Difference Systems. Among the results, it was evident how those municipalities, with different politicalinstitutional contexts, converge in similar relationships and actors to guarantee public purchases in school feeding. In spite of convergence, it was also possible to observe differences in the role of the State and its policies, which was stronger and with greater trajectory in Brazil than in Colombia; international cooperation, which was stronger and more important in Colombia than in Brazil; the role of social actors, which was strong and organized in both municipalities but with greater institutional support in Brazil than in Colombia; and, finally, the leadership of different actors and local agents to implement school feeding that include peasant and family farmers.
\end{abstract}

Keywords: Public Purchases. School Meal. Policy Networks. Comparative Politics. Brazil. Colombia.

\section{Atores, redes e alimentos: a participação da agricultura familiar e campesina na alimentação escolar do Brasil e da Colômbia}

\section{Resumo}

O artigo tem como objetivo comparar as redes de compras públicas de produtos da agricultura familiar e campesina para os programas de alimentação escolar nos municípios de Granada (Antioquia-Colômbia) e São Lourenço do Sul (Rio Grande do Sul-Brasil) nos anos de 2016 e 2017. Para tal fim, o estudo partiu da abordagem teórica metodológica de redes de política pública, articulada às abordagens de Análise de Redes Sociais e comparação de Sistemas de Máxima Diferença. Entre os resultados obtidos se evidenciou como os municípios, com contextos políticoinstitucionais diferenciados, convergem em relações e atores semelhantes para garantir compras públicas para a alimentação escolar. Em que pese à convergência, também foi possível observar diferenças quanto ao papel do Estado e suas políticas, mais fortes e com maior trajetória no Brasil que na Colômbia; a cooperação internacional, mais forte e importante na Colômbia que no Brasil; o papel dos atores sociais, fortes e organizados em ambos os municípios, mas com maior suporte institucional no Brasil que na Colômbia; e, finalmente, liderança de diferentes atores e agentes locais para implementar a participação da agricultura familiar na alimentação escolar.

Palavras chave: Alimentação Escolar. Redes de Políticas Públicas. Política Comparada. Brasil. Colômbia.

\section{Introducción}

La soberanía y seguridad alimentaria y nutricional (SSAN) ha recobrado un renovado valor en América Latina como resultado de las reivindicaciones de los movimientos sociales a causa de las crisis de alimentos desatadas en los últimos años (OCARIZ, 2015; ROMERO, 2012; RUBIO, 2011) y el incremento en la importación de alimentos estimulada por los tratados de libre comercio (LÓPEZ, 2012). A su vez, y según afirman Maluf, Schmitt y Grisa (2009), esta reciente preocupación también se 
asocia al avance de la commoditización de las economías, que ha dejado, como resultado, la dependencia alimentaria y sus efectos degradadores sobre la salud y el medio ambiente. Bajo este panorama, la alimentación escolar pasó de ser un programa público de rendimiento y/o permanencia escolar, para transformarse en un instrumento multitask al garantizar al mismo tiempo un mayor y mejor acceso a alimentos saludables, aumentar la renta de campesinos y agricultores familiares y dinamizar economías locales.

En Colombia, las compras públicas de la agricultura campesina, familiar y comunitaria para el Programa de Alimentación Escolar (PAE) fueron promovidas desde el Instituto Colombiano de Bienestar Familiar (ICBF), quien a través de la Estrategia Compras Locales lideró la compra directa a campesinos en los municipios. Posterior a ello, mediante el Decreto 1852 de 2015 (COLOMBIA, 2015a), éstas pasan a formar parte del Ministerio de Educación Nacional (MEN) al exigir del operador del PAE la adquisición del $20 \%$ en alimentos a productores locales y reafirmar la descentralización total del programa en departamentos y municipios. Aunque poco clara esta configuración, las compras se estructuran desde las secretarías de educación, las secretarías de desarrollo económico (o quien haga sus veces) y participan mediante ruedas de negocios las asociaciones de productores y los operadores del programa.

En Brasil, la institucionalización de estas compras se realizó vía Ley federal 11.947 de 2009 (BRASIL, 2009a), que regula el Programa Nacional de Alimentación Escolar (PNAE) y define la adquisición de mínimo el 30\% de alimentos provenientes de la agricultura familiar mediante los recursos que son transferidos al municipio a través del Fundo Nacional de Desenvolvimento da Educação (FNDE). El programa funciona descentralizado en municipios y estados y, en ocasiones, es ejecutado de forma directa por las escuelas. En general, estas compras se estructuran sobre la base de cuatro actores claves: los agricultores familiares, el sector de la alimentación escolar adscrito a la secretaría de educación (o quien haga sus veces), los órganos de asistencia técnica y el Conselho de Alimentação Escolar (CAE) quien realiza el control social.

Lo anterior nos lleva a pensar y analizar este proceso político en clave de redes de política pública. Visto en perspectiva de redes, las compras públicas se derivan, no de la formalidad de una ley o norma, sino más bien, de las articulaciones entre actores y agentes en la formulación o implementación del programa en un contexto determinado (KLIJN, 1998; PAULILLO; ALMEIDA, 2011; PORRAS, 2001; ZURBRIGGUEN, 2004). Siguiendo esta línea analítica, estos actores y agentes están dotados de intereses y recursos y su acción está mediada por reglas formales e informales.

De esta forma, las redes obedecen a ese conjunto de intercambios, en el marco del proceso político de las compras públicas, que vinculan entre sí actores en distintas escalas territoriales (local, regional, departamental/estadual, federal/nacional, internacional). A su vez, estas compras denotan una especificidad al ser, un instrumento de desarrollo rural para el acceso a mercados vinculado a un programa social, la alimentación escolar. 
Para llegar a este propósito analítico, esta investigación integró dos abordajes metodológicos ${ }^{1}$, el Análisis de Redes Sociales (ARS) y la política comparada mediante el Diseño de Sistemas de Máxima Diferencia (DSMD). Desde el ARS se pretende mapear los actores y agentes que participan del proceso político y sus niveles de actuación. Por su parte, el DSMD propone evaluar un resultado político común, como en este análisis (la inserción de las compras públicas a campesinos y agricultores familiares), a partir de sus rasgos diferenciados.

En consecuencia, este artículo se propone analizar de forma comparada cómo dos municipios de países con dinámicas políticas diferenciadas llegaron a un mismo resultado político: las compras públicas de campesinos y agricultores familiares para la alimentación escolar mediante el análisis de los actores, los elementos que circulan por la red (reglas, recursos e intereses) y las relaciones (cooperación y conflicto) en el período 2012-2016.

\section{Contexto municipal alimentación escolar en Granada/Colombia y São Lourenço do Sul/Brasil}

El municipio de Granada (Antioquia-Colombia), nuestro primer caso de estudio, constituye una localidad pionera en la implementación de las compras locales de campesinos desde el año 2008. Posteriormente, en 2015, fue piloto para diseñar un programa de compras públicas en el país, proyecto desarrollado en alianza con la Organización de las Naciones Unidas para la Alimentación y la Agricultura (FAO)-Embajada de Brasil. Se considera un caso relevante, en materia de compras públicas y alimentación escolar, al lograr que por primera vez una organización de campesinas y campesinos operara el PAE municipal en 2014 y al priorizar, en el marco de este proceso, la compra directa de la producción de los agricultores locales. Este municipio está localizado en el departamento de Antioquia, en la región del oriente antioqueño, territorio constituido por 22 municipios más. Así, Granada tiene una extensión territorial de 195 km2 y, según el Plan Municipal de Desarrollo 2016-2019, se calcula una población al 2015 de 9.858 habitantes (ALCALDÍA DE GRANADA, 2016), con una cobertura neta en educación de $78,24 \%$ (COLOMBIA, 2018) y un promedio anual de matrícula en el período analizado de 1.648 estudiantes (ver tabla 1).

\footnotetext{
${ }^{1}$ Fueron realizadas 36 entrevistas semiestructuradas, 4 grupos focales y 31 encuestas de redes en Colombia y Brasil en el período de Juno de 2016 a octubre de 2017.
} 
Tabla 1. Principales características de la adquisición de alimentos a campesinos y agricultores familiares en los programas de alimentación escolar de São Lourenço do Sul (RS-Brasil) y Granada (Antioquia-Colombia)

\begin{tabular}{|c|c|c|c|c|c|c|}
\hline $\begin{array}{c}\text { País/ } \\
\text { municipio }\end{array}$ & $\begin{array}{l}\text { Entidad } \\
\text { responsable }\end{array}$ & Proceso licitatorio & $\begin{array}{c}\text { Cobertura del } \\
\text { programa }\end{array}$ & $\begin{array}{l}\text { Compras a los } \\
\text { campesinos/ag } \\
\text { ricultores } \\
\text { familiares }\end{array}$ & $\begin{array}{c}\text { Promedio de } \\
\text { estudiantes/mu } \\
\text { nicipio } \\
(2012-2016)\end{array}$ & $\begin{array}{l}\text { Promedio } \\
\text { anual de } \\
\text { recursos } \\
\text { transferidos } \\
\text { del PAE al } \\
\text { municipio en } \\
\text { USD } \\
(2012-2016)\end{array}$ \\
\hline $\begin{array}{l}\text { Brasil/ São } \\
\text { Lourenço } \\
\text { do Sul } \\
\text { (RS) }\end{array}$ & $\begin{array}{l}\text { FNDE } \\
\text { (federal)/ } \\
\text { Setor da } \\
\text { alimentação } \\
\text { escolar } \\
\text { (municipal) }\end{array}$ & $\begin{array}{l}\text { No. Vía chamada } \\
\text { pública son } \\
\text { adquiridos los } \\
\text { alimentos } \\
\text { directamente de los } \\
\text { agricultores } \\
\text { familiares. Art. } 4 . \\
\text { Ley } 11.947 \text { de } 2009 \text {. } \\
\text { En las compras } \\
\text { convencionales, } \\
\text { que no son de la } \\
\text { agricultura familiar, } \\
\text { es realizada vía } \\
\text { licitación. }\end{array}$ & $\begin{array}{l}\text { Universal. } \\
\text { Aplicable a } \\
\text { toda la } \\
\text { población } \\
\text { escolar, } \\
\text { incluso, } \\
\text { población } \\
\text { extra-edad. }\end{array}$ & $\begin{array}{l}\text { Realizadas por } \\
\text { el sector de la } \\
\text { alimentación } \\
\text { escolar. }\end{array}$ & 6.907 & 131.755 \\
\hline $\begin{array}{l}\text { Colombia/ } \\
\text { Granada } \\
\text { (Ant) }\end{array}$ & $\begin{array}{l}\text { MEN } \\
\text { (nacional)/ } \\
\text { oficina de } \\
\text { complementa } \\
\text { ción } \\
\text { alimentaria } \\
\text { (municipal) }\end{array}$ & $\begin{array}{l}\text { Si. Vía contratación } \\
\text { del operador PAE. } \\
\text { Ley } 80 \text { de } 1993 \text { (Ley } \\
\text { de contratación } \\
\text { pública). }\end{array}$ & $\begin{array}{l}\text { Focalizada en } \\
\text { población } \\
\text { escolar de baja } \\
\text { renta y de } \\
\text { educación } \\
\text { básica } \\
\text { primaria. Sin } \\
\text { embargo, } \\
\text { debido a la } \\
\text { gestión } \\
\text { municipal } \\
\text { Granada } \\
\text { cuenta con } \\
\text { cobertura } \\
\text { universal. }\end{array}$ & $\begin{array}{l}\text { Realizadas por } \\
\text { el operador del } \\
\text { programa. Se } \\
\text { promueven } \\
\text { cooperativas } \\
\text { como } \\
\text { operadores. }\end{array}$ & 1.648 & 115.568 \\
\hline
\end{tabular}

Fuente: Elaboración propia con base en entrevistas

Según el perfil productivo del municipio, entre los principales productos agropecuarios se encuentran: café, caña, frijol, papa, tomate y mora. Aunque también es posible encontrar producción de frutales a pequeña escala como: uchuva, lulo, tomate de árbol, granadilla, maracuyá, piña, papaya, aguacate y cítricos. La ganadería doble propósito, avicultura, porcicultura y piscicultura también conforman el sistema pecuario municipal. Según este mismo documento, el 91,62\% del territorio se ubica en el microfundio y minifundio (PROGRAMA DE LAS NACIONES UNIDAS PARA EL DESARROLLO (PNUD), 2015).

Por su parte, el municipio de São Lourenço do Sul (Rio Grande do Sul, Brasil), nuestro segundo caso de estudio, es considerado uno de los municipios exitosos en la implementación de las compras públicas de la agricultura familiar para el PNAE por lograr, en 2015, adquirir géneros alimenticios de los agricultores familiares con el 99,7\% de los recursos transferidos por el FNDE, cuando la ley 11.947 de 2009 sugiere mínimo 30\%. Cuenta además con un sector específico dentro de la alcaldía que coordina la alimentación escolar. 
Este municipio está localizado al suroriente del estado de Rio Grande del Sur, último estado al sur de Brasil. Con una extensión territorial de 2.031 km2 y al 2016 contaba con una población, según estimaciones del Instituto Brasileiro de Geografia e Estatística (IBGE), de 44.561, con una tasa de escolarización de 97,6\% (BRASIL, 2016) y un promedio de 6.907 matriculados en el período estudiado (ver tabla 1). Entre los principales productos agropecuarios del municipio se encuentran lácteos, maíz, frijol, soya, arroz, tubérculos, cebolla, espárragos, pimienta, ajo y maní, además de la cría de suinos y bovinos. En relación a la distribución de la tierra el $95 \%$ es ocupada por pequeños y medianos agricultores y una parte importante de la población se dedica al cultivo del tabaco y el turismo (COUTO, 2012, p. 19).

Como se observó en la tabla 1, ambos contextos municipales poseen características disímiles en cuanto temporalidad, procesos licitatorios, recursos trasferidos, número de estudiantes, entre otros aspectos relevantes. Sin embargo, predominó ser pioneros municipales en la inserción de las compras directas a campesinos y agricultores familiares en los programas de alimentación escolar. En este sentido, la convergencia de este proceso político se expresa en los actores y agentes locales y sus relaciones para incentivar las compras públicas a campesinos y agricultores familiares en los PAE como se demostrará en los apartados posteriores.

\section{Mapeo de actores comparado}

Al comparar ambos casos, tanto en Granada, como en São Lourenço do Sul, existen semejanzas en los actores que participan del proceso de compras públicas para la alimentación escolar. El sector cooperativo², las ONG's, la cooperación internacional y las alcaldías o sus sectores, entre otros. Esto puede ser explicado, de forma parcial, en función de la transferencia de políticas e instituciones desde Brasil hacia Colombia, proceso realizado, mediante intercambios técnicos entre funcionarios de los distintos ministerios (educación y agricultura) y algunas gobernaciones. De forma preliminar y una vez comparados los contextos, se puede afirmar que el modelo brasilero está implícito en los cambios ocurridos en las instituciones de la alimentación escolar nacional colombiana, a partir del año 2013, en los siguientes aspectos: 1) descentralización del PAE en municipios y departamentos; 2) control social mediante la creación de Consejos de Alimentación Escolar (CAE); 3) creación de un fondo de recursos común para la financiación del programa; 4) presencia del sector cooperativo y/o solidario; 5) instrumento de compras públicas a productores locales.

\footnotetext{
${ }^{2}$ Se ha querido resaltar la dualidad que comporta el sector cooperativo o solidario como agente económico que se guía por principios sociales, es decir, como actor social también. Para tal fin se representó mediante cuadrados en las ilustraciones 1 y 2.
} 
Ilustración 1. Mapeo de actores Granada (Antioquia-Colombia)

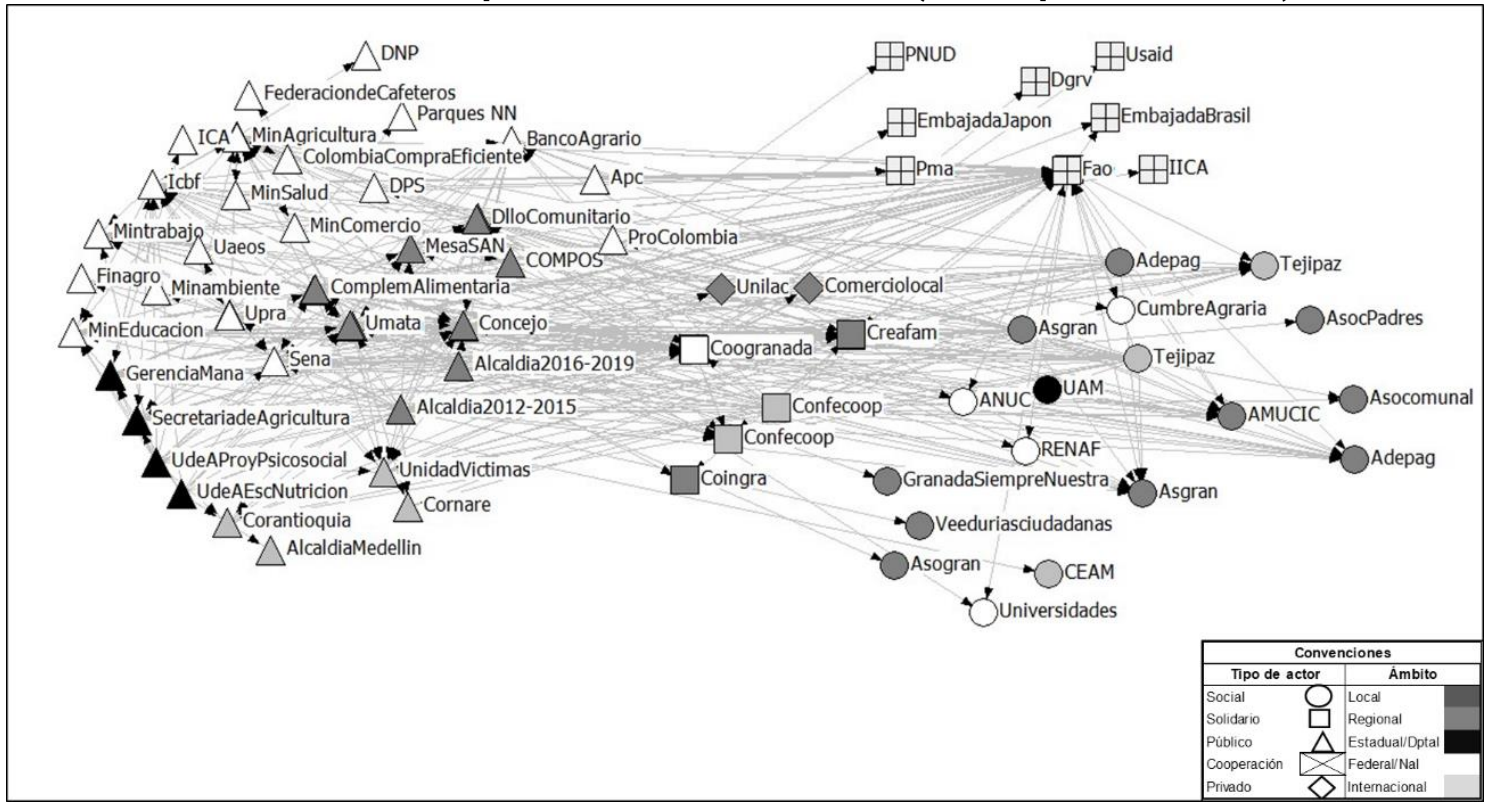

Fuente: Elaboración propia mediante Ucinet

De forma específica, el Estado 3 difiere en mayor medida en ambos casos, tanto por la trayectoria, mayor en Brasil, que en Colombia; como por las capacidades institucionales de sus organizaciones, más maduras en Brasil que en Colombia, en asuntos claves como: a) instituciones específicas y con funciones definidas, b) asistencia técnica tanto a gestores públicos y ciudadanos, como, a agricultores familiares y c) agregación de demanda mediante la compra de producción a cargo de la compañía estatal de abastecimiento CONAB.

En relación a los actores sociales ${ }^{4}$ (ver ilustraciones 1 y 2 ) se puede afirmar que, en ambos casos, existe una agricultura familiar (São Lourenço do Sul) o campesina (Granada) fuerte y organizada, ya sea mediante asociaciones de productores (Granada), o grupos para la producción (São Lourenço do Sul), que a su vez están adscritos a otras organizaciones de comercialización Gransol (Granada), Sul Ecológica (São Lourenço do Sul). En ambos casos, las ONG que participan tienen capacidad de incidencia política, aunque, varían en función del ámbito donde la ejercen. De un lado, la ONG Tejipaz 5 (Granada), se relaciona con otros actores no locales, por ejemplo, Alcaldía de Medellín y FAO. De otro lado, la ONG CAPA ${ }^{6}$ (São Lourenço do Sul), se circunscribe a un plano más local-regional, aunque con relaciones internacionales, por ejemplo, a través de Pão Para o Mundo (PPM).

\footnotetext{
3 Identificado con triángulos en las ilustraciones 1 y 2.

4 Identificados con círculos en las ilustraciones 1 y 2.

5 Tejiendo territorio para la paz.

${ }^{6}$ Centro de Apoio e Promoção da Agroecologia.
} 
Ilustración 2. Mapeo actores São Lourenço do Sul (Rio Grande do Sul-Brasil)

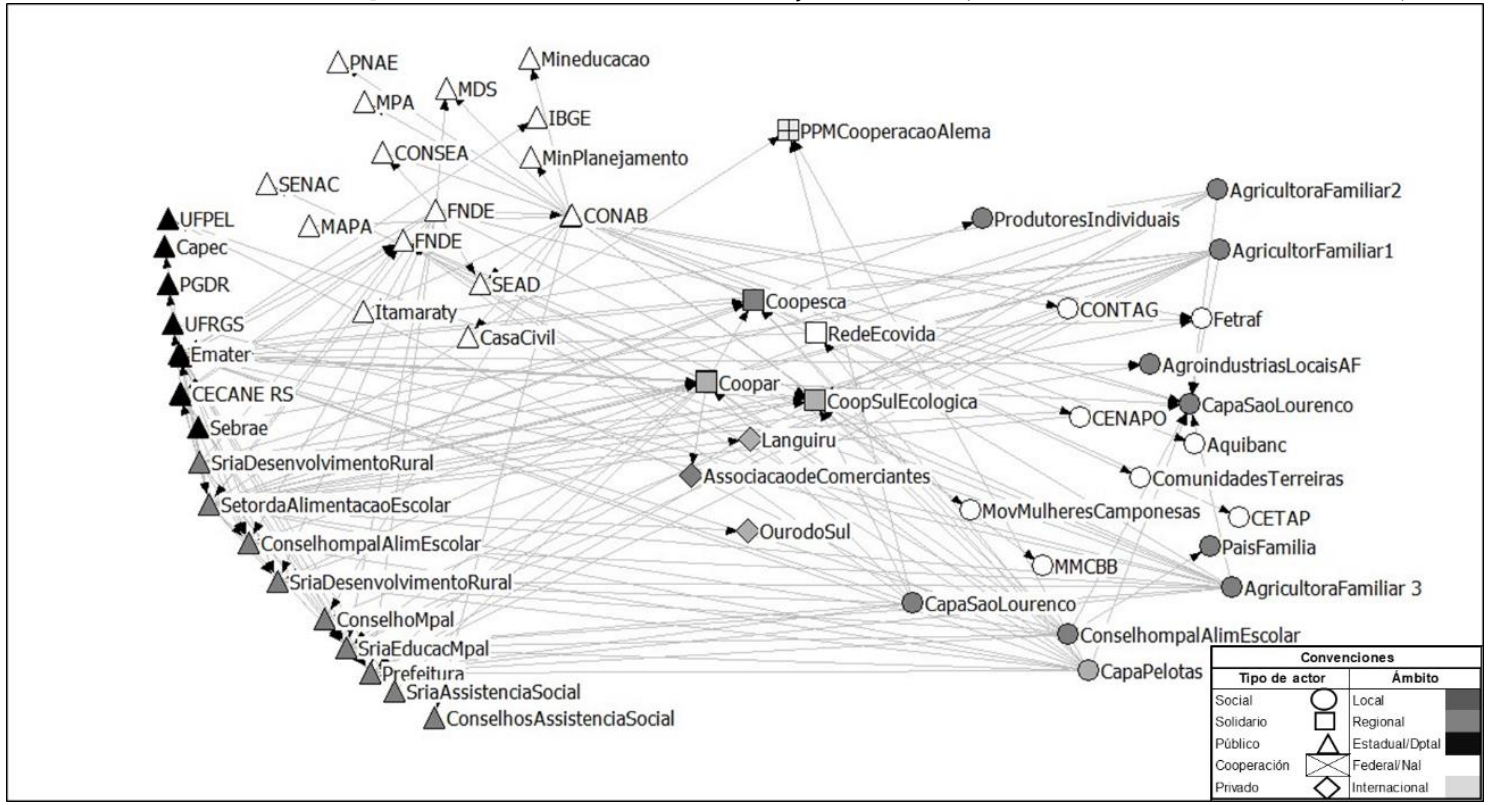

Fuente: Elaboración propia mediante Ucinet

En cuanto a los agentes económicos, en ambos casos existe una alta centralidad del sector cooperativo. Esta importancia puede ser valorada en función de la particularidad de la agricultura familiar y campesina que demanda procesos de comercialización diferenciados, que solo el sector solidario y asociativo, puede construir. Ésta es la principal forma organizativa para la comercialización en ambos municipios. No obstante, difieren en uno y otro caso, tanto por el surgimiento como por el carácter de la cooperativa. Es así como en el caso de Granada las cooperativas son financieras $y$, por lo tanto, se integraron al garantizar el tema del crédito al productor, posteriormente y de forma reciente se concentran, tanto en la asistencia técnica, como en la comercialización en red. En contraste, en el caso de São Lourenço do Sul, las cooperativas fueron el resultado de la producción pues al tener excedentes o una producción diferenciada los agricultores no contaban con canales de acceso adecuados para la comercialización de sus productos.

Para finalizar, aunque en ambos municipios existe presencia de agencias internacionales, la cooperación internacional ${ }^{7}$ difiere en gran medida en función de la cantidad, mayor número en el caso de Granada, por ejemplo, con organizaciones como la Confederación Alemana de Cooperativas (DGRV), Agencia de los Estados Unidos para el Desarrollo Internacional (USAID), el Programa Mundial de Alimentos (PMA), Embajadas del Brasil y de Japón, Programa de las Naciones Unidas para el Desarrollo (PNUD), Instituto Interamericano de Cooperación para la Agricultura (IICA) y FAO. De forma destacada, agencias de cooperación como DGRV (alemana) y USAID (estadounidense), además del financiamiento vía proyectos, inciden en las metodologías empleadas en el trabajo con campesinos del municipio. En contraste en São Lourenço do Sul, PPM cooperante alemán que participa mediante la financiación de proyectos del CAPA. Su incidencia es baja en cuanto a la exigencia de metodologías específicas y, por el contrario, se centra en estimular la producción de alimentos limpios y la generación de renta para los agricultores en el municipio.

\footnotetext{
7 Ver figura cuadrado con diagonales en su interior en las ilustraciones 1 y 2.
} 
Actores, redes y alimentos: la participación de la agricultura familiar y campesina en la

alimentación escolar del Brasil y Colombia

\section{Elementos que circulan por la red de actores: reglas, recursos e intereses}

\subsection{Reglas formales e informales}

Las reglas institucionales, tanto formales, como informales, cumplen en ambos casos una función común: incorporar alimentos provenientes de la agricultura campesina y familiar en el programa de alimentación escolar. Este objetivo requirió de ajustes o creación de otras normas o reglamentos que flexibilizaran o modificaran los criterios del proceso de compras públicas y/o contratación pública.

En el contexto brasilero, las compras públicas se rigen bajo la ley de licitaciones 8.666 de 1993 (BRASIL, 1993). Esta implica la compra bajo el criterio del menor precio y, con lo cual se estimulaba fundamentalmente que el mismo gran intermediario ganase las licitaciones, dada su capacidad de acopiar productos para vender a más bajo precio. Por su parte en Colombia, más que la ley de licitaciones, aplica la Ley 80 de 1993 (COLOMBIA, 1993) que obliga al Estado a subcontratar con terceros la operación de los programas, sean estos sociales o no, ley que estimuló una gran cadena de intermediarios al interior del Estado y fuera de él, y que, en el contexto específico de la alimentación escolar, llegó a pasar hasta por seis grandes agentes (entre funcionarios y operadores) antes de llegar al beneficiario final.

Así, reorganizar el programa e incluir las compras a la agricultura campesina y familiar, llevó a formular en 2009 en Brasil y en 2015 en Colombia nuevas normas institucionales. En el contexto brasilero, la ley 11.947 que en su artículo 4 instituye las compras públicas a la agricultura familiar. En el contexto colombiano, el decreto presidencial 1852 que reestructura el PAE, reafirma su paso del ICBF al MEN e instituye la descentralización del programa en municipios y departamentos para, de esta forma, estimular las compras más cercanas a los territorios. Ambas, ley y decreto, fueron posteriormente desarrolladas en las resoluciones 38 de 2009, 26 de 2013 y 4 de 2015 (BRASIL, 2009b, 2013, 2015) en el contexto brasilero; y en la resolución 16432 de 2015 (COLOMBIA, 2015b) en el contexto colombiano. Aunque estas resoluciones tuvieron objetivos diferentes, demuestra la necesidad de ajuste periódico a las reglas institucionales, y según el FNDE, estas normas permiten revisar y modificar el programa a medida que éste avanza y de ir institucionalizando prácticas y aclarando dudas que van surgiendo en el camino de la implementación.

Se observó también, en el contexto colombiano, la construcción de reglas en el escenario municipal. De esta forma, el alcalde de Granada, mediante decreto municipal 039 de 2015, institucionalizó las compras públicas al interior del COMPOS $^{8}$, con la creación de la mesa de agricultura familiar y compras públicas, que a su vez diseña el mecanismo e instituye los actores que participan de la concertación de las compras públicas al ser la alimentación escolar parte importante de estas. Aunque en el caso de São Lourenço do Sul no existe tal formalidad, se observa sí, que emergen prácticas, configurando reglas informales, en la interacción cotidiana de los actores que participan de las compras para la alimentación escolar. Por ejemplo, se ha concertado entre los actores que al existir varios proveedores para un mismo producto se divide en cantidades iguales para todos, en vez de realizar un sorteo, como sugiere la ley. También se estudia en detalle antes de incluir un nuevo producto

\footnotetext{
${ }^{8}$ Consejo Municipal de Política Social.
} 
en la chamada pública si este efectivamente beneficiará a las agroindustrias de la agricultura familiar.

\subsection{Recursos actores y agentes}

Los recursos, son mediadores de la capacidad de influencia de un actor en la red. Esta capacidad también está determinada por el carácter misional de las entidades, como sucede, de forma específica, con las organizaciones del Estado. En este sentido, un mapeo de recursos de los actores institucionales arrojó como resultado la variedad de los mismos y su uso de uno u otro en función de la situación. Incide también el contexto político institucional de las organizaciones, pues este repercute en la pérdida o desarticulación de programas del Estado, aunque estos hayan contado con una amplia trayectoria.

Así, la asistencia técnica compone el primero de esos recursos. Ésta va desde el proceso de acompañamiento directo a los productores, con un fuerte énfasis en temas agropecuarios y administrativos que es realizado por actores específicos como FAO, UMATA ${ }^{9}$, Gerencia Maná, UdeA (departamento de trabajo social), en el caso de Granada; y EMATER ${ }^{10}$ y SEAD ${ }^{11}$, en el caso de São Lourenço do Sul. Un segundo recurso el financiero, del cual se deriva el funcionamiento adecuado del programa en ambos municipios. Este es transferido de forma directa al municipio por el FNDE, quien también complementa el recurso, en el caso de São Lourenço do Sul y en Granada este recurso se transfiere desde el MEN a la Gobernación de Antioquia para luego ser complementado y desembolsado al municipio.

Un tercer recurso, el control y vigilancia, realizado a las entidades ejecutoras del PAE. En el caso de São Lourenço do Sul, el control y vigilancia es ejercido por el FNDE a través de los CAE, mediante el diligenciamiento de un formulario que atiende a las preguntas de eficiencia en la utilización del recurso, la contrapartida presupuestal, el suministro de la alimentación durante los 200 días lectivos y la forma como se da la participación de la agricultura familiar, entre otros, a su vez, y de forma eventual se realizan visitas por parte del FNDE a los municipios como sucedió en São Lourenço do Sul en los años 2011 y 2016.

Por el contrario, en Granada, el control y vigilancia es realizado por un sinnúmero de actores. Desde la Gerencia de Maná, la Universidad de Antioquia a través de delegación de competencias por parte de la gerencia, por la secretaria de gobierno del municipio, entre otros. A diferencia de São Lourenço do Sul, el papel de los actores sociales es bastante débil en el proceso. Siendo los padres de familia el actor más visible, pero con baja incidencia ante las organizaciones del Estado. El control y vigilancia, como recurso de poder, es ejercido para definir los ciclos de menú, de arriba hacia abajo y, el cumplimiento de requisitos legales y administrativos que desconocen las capacidades de los territorios. Entre el 2012 y el 2015 fue usado con mayor vehemencia para justificar la necesidad de compras en pocos operadores, que no admitían la inclusión de productos locales por no contar con tablas nutricionales y registros ante el Instituto Nacional de Vigilancia de Medicamentos y Alimentos (INVIMA).

\footnotetext{
9 Unidad Municipal de Asistencia Técnica.

${ }^{10}$ Empresa de Assistência Técnica e Extensão Rural.

${ }^{11}$ Secretaria Especial de Agricultura familiar e do Desenvolvimento Agrário.
} 
Por su parte, los actores sociales cuentan con un recurso estratégico para el PAE/PNAE: la organización social de la producción y la comercialización. En el caso de São Lourenço do Sul, su tradición proviene del estímulo de las ONG y con apoyo de la cooperación internacional, al contribuir en la organización de la producción y la creación de cooperativas para la comercialización. En el caso de Granada, la trayectoria histórica que tiene la región en la producción de alimentos durante la revolución verde y la fuerte tradición en organización social de base comunitaria. Este recurso se transformó en plataforma para el proceso, al verse fortalecido y ampliado con la llegada del piloto FAO-Embajada de Brasil y con ello, abriendo mercados para los campesinos.

Si quisiéramos resumir el principal recurso de los agentes económicos en las compras públicas diríamos: economía solidaria, o, dicho en otras palabras, en todo mercado debe existir un lucro, pero éste debe ser en sus justas dimensiones. Por ello, no es casualidad que las cooperativas sean el actor principal de las compras públicas. Esto sucede porque este proceso político requiere de unas condiciones específicas en los actores del mercado, que cumplan una función social en el territorio y no solo se lucren, que tengan una sensibilidad y credibilidad en la importancia del papel de los agricultores en la alimentación, sea escolar o no, pero sobretodo y de forma enfática, que sus requisitos se adecuen a las condiciones específicas de las y los pequeños productores.

Para finalizar, a medida que la investigación avanzó fue evidente el liderazgo de las mujeres en el proceso, al ser un recurso importante en la consolidación del PAE/PNAE en el ámbito municipal. Este liderazgo, como recurso de las compras públicas, se configura por el hecho de ser mujeres quienes ocupan u ocuparon los principales cargos de dirección o liderazgo, sea como gestoras de las políticas, sea en las organizaciones de productores, en las cooperativas o en las ONG como se observa en la tabla 2.

Tabla 2. Mujeres lideresas del proceso de compras públicas en la alimentación escolar

\begin{tabular}{|c|c|c|}
\hline País & Entidad & Cargo \\
\hline Brasil & Cooperativa Sul Ecológica & Integrante de la dirección \\
\hline Brasil & CAPA & Coordinadora \\
\hline & & Coordinadora División \\
\hline Brasil & FNDE & $\begin{array}{l}\text { Agricultura Familiar, } \\
\text { consultoras }\end{array}$ \\
\hline Brasil & $\begin{array}{l}\text { Setor da alimentação escolar, } \\
\text { São Lourenço do Sul }\end{array}$ & $\begin{array}{l}\text { Coordinadora, nutricionista, } \\
\text { gestora }\end{array}$ \\
\hline Brasil & Emater, São Lourenço do Sul & Gestoras sociales \\
\hline Brasil & CECANE & Coordinadora de gestión \\
\hline Colombia & Coogranada & $\begin{array}{l}\text { Gestora de proyectos } \\
\text { sociales }\end{array}$ \\
\hline Colombia & Tejipaz & $\begin{array}{l}\text { Directora, zootecnista (entre } \\
\text { otras) }\end{array}$ \\
\hline Colombia & UdeA-Trabajo social & $\begin{array}{l}\text { Coordinadora proyecto } \\
\text { atención psicosocial }\end{array}$ \\
\hline Colombia & Alcaldía municipal Granada & $\begin{array}{l}\text { Secretaria de gobierno 2012- } \\
2015\end{array}$ \\
\hline Colombia & $\begin{array}{l}\text { Of. De complementación } \\
\text { alimentaria }\end{array}$ & $\begin{array}{l}\text { Contratista y responsable de } \\
\text { la oficina }\end{array}$ \\
\hline
\end{tabular}

Fuente: Elaboración propia. 


\subsection{Recursos actores y agentes}

Son diversos los intereses que motivan a los actores a participar en la red tanto en el municipio de Granada, como en São Lourenço do Sul. Estos van desde temas estructurales como la construcción de políticas públicas y limitar la gran cadena de intermediarios, pasando por el simple cumplimiento normativo, hasta llegar a aspectos más concretos como la calidad de la producción.

En el caso de Granada, existen dos intereses específicos que motivan a los actores locales a participar del proceso de compras públicas. El primero de ellos y, de un peso significativo por el momento que vive el país, el proceso de construcción de paz. Este interés está mediado por los acuerdos de paz que ya fueron aprobados en el país y que pasaron a tener un rango jurídico constitucional. Las compras públicas en este sentido se consideran la ruta más eficaz para garantizar la dinamización de las economías de los municipios más afectados por el conflicto armado y, a su vez, como estrategia económica para apoyar la reinserción de los excombatientes a la vida civil. En este caso, el sector solidario y cooperativo es el actor clave del proceso. El segundo de ellos, desde el punto de vista formal, el interés del MEN por la permanencia escolar de los estudiantes, fin que se espera lograr con una adecuada alimentación en la escuela.

En el caso de São Lourenço do Sul, fundamental es la preocupación por mejorar la calidad de los alimentos que son entregados en la alimentación escolar y contribuir a mejorar las condiciones de vida de los agricultores familiares. El estímulo a la compra de alimentos locales garantizó tener alimentos frescos siempre, mucho más diversificados e incluso con pequeños procesos de industrialización al tiempo que mejoraban los ingresos de los agricultores. Esta calidad se sigue estimulando por la vía de ampliar el número de proveedores, esto implica que los agricultores deban estar en progreso y evolución constante, y, por lo tanto, evitar que el proceso se estanque.

\section{Análisis relacional comparado: cooperación y conflicto PAE Granada y PNAE São Lourenço do Sul}

Observamos en apartados anteriores que en el proceso de compras públicas convergen actores sociales e institucionales, agentes económicos y cooperación internacional, quienes a su vez participan del proceso de compras públicas según intereses, recursos y reglas institucionales que median esta interacción y se intercambian en la relación. En consecuencia, este trabajo exploró dos tipos de relaciones, la cooperación y el conflicto, para conocer en qué temáticas existe un mayor consenso y cuáles son los asuntos que despiertan disenso entre actores y agentes, así como, los mecanismos para abordar y desarrollar estas situaciones, como se describe a continuación.

\subsection{Cercanía, confianza y negociación como formas de cooperación}

La cooperación se ha denominado una primera forma de interacción en esta red. Al momento de preguntar a los entrevistados por la forma en que ellos cooperan con otros actores para la ejecución de las compras públicas referencia a tres 
conceptos: cercanía (tanto geográfica, como relacional), confianza y negociación. Sin embargo, el tema de negociación es mucho más fuerte en São Lourenço do Sul, mientras que la confianza es clave en Granada, en ambos casos la cercanía o proximidade constituye una forma importante de relacionarse.

Veamos, cómo ocurre la negociación en el caso de São Lourenço do Sul. Para los entrevistados, la negociación constituye una relación directa con el agricultor, que incluye consensuar y pactar con ellos temas sensibles como la chamada pública, días de entrega de los alimentos, períodos de cosecha y el intercambio de productos frente a la carencia de uno u otro. Aunque en el caso de Granada, algunas negociaciones también se dieron en el ámbito local, gran parte de éstas ocurrieron en escenarios departamentales y hasta nacionales. En parte, esta configuración se dio por el hecho de ser un proceso emergente y piloto que no cuenta con una trayectoria tan amplia como en el caso de São Lourenço do Sul, así como, a causa de la mediación de FAO en materia de compras públicas.

Una segunda forma de cooperación es la confianza. Este es el resultado de un período más o menos estable en la relación y, en especial, en el caso de Granada se trató de un mecanismo de reconstrucción del tejido social y una apuesta institucional bastante fuerte. Por lo tanto, la confianza se edificó a partir de definir reglas claras en conjunto, por ejemplo, instituir un único precio al definir un mismo valor por alimento y su validación periódica por los actores; con la participación de actores que dieran soporte al proceso como la alcaldía municipal y la FAO; a través de la mediación de la Universidad en proyectos de extensión que contribuían a reconstruir el tejido social y restablecer la confianza entre vecinos.

Además de estas dos formas de cooperación, existe también en el proceso de compras públicas una relación mucho más cercana entre los actores. En el caso de Granada y São Lourenço do Sul, esta proximidad es el resultado de la descentralización del PAE, que permite a los actores locales construir un vínculo más directo con el operador del programa (caso Granada). Para otros, la proximidad se ve reflejada en tener un diálogo mucho más abierto, honesto y rápido al momento de resolver problemas cotidianos en la implementación de las compras a los agricultores (caso São Lourenço do Sul).

\subsection{Las distintas caras del conflicto}

El conflicto constituye una segunda forma de interacción en la red. Este tipo de relación implica la disputa por el acceso a recursos escasos (BOBBIO; MATEUCCl; PASQUINO, 1998, p. 225). Como disputa, en el marco del proceso de las compras públicas para la alimentación escolar, se evidencia en quién y cómo se construyen las reglas de juego y se accede a recursos. Así, un aspecto importante es el ámbito donde se toman esas decisiones, es decir, si es local, estadual/departamental y/o federal/nacional y quiénes son los actores y agentes que las definen. Como veremos en las siguientes páginas, existen conflictos comunes a los dos municipios y otros que son específicos. Algunos de estos conflictos comparten el hecho que cuando en un caso es motivo de disputas, por ejemplo, el precio, en el otro caso es motivo de cooperación, como en el caso de los intermediarios.

De esta forma, el cambio de gobierno como aspecto que genera conflictos, es común a ambos municipios. Se entiende además que incide, tanto, cuando se trata 
del gobierno municipal, como, del gobierno departamental/estadual y/o nacional/federal. El conflicto se genera al momento de poner en vilo políticas en curso, como reglas y procesos de amplia trayectoria, como en el caso de São Lourenço do Sul, o de desestabilizar los aspectos ya avanzados y construidos como en el caso de Granada. En específico, en el contexto brasilero existe una mayor preocupación por la pérdida acelerada de espacios para la agricultura familiar en general, por ejemplo, reformas al régimen de seguridad social de los agricultores, debilitamiento del PAA ${ }^{12}$, entre otros.

Otra cuestión que genera conflictos obedece al monto de recursos transferidos. En el caso de São Lourenço do Sul, el valor que es transferido por el FNDE no aumenta de forma regular o su incremento es mínimo si se le compara con las necesidades. En la actualidad es transferido un valor de 0.36 centavos (BRL). En el caso de Granada, aun no existe un fondo común y los recursos son transferidos a la gobernación de Antioquia, complementados por gobernación, alcaldía y cooperativas, siendo éste alrededor de \$1.130 (COP). Sin embargo, dada la cadena de intermediarios por la que pasa, este valor se ve reducido de forma significativa.

Una tercera y última cuestión en común frente a las relaciones de conflicto está relacionada con las merendeiras o manipuladoras de alimentos. Al inicio del programa en el caso de São Lourenço do Sul, el proceso de preparación de alimentos pasó por sinnúmeros desafíos, el más visible, la negativa a preparar alimentos in situ por parte de las merendeiras debido a dos factores, de un lado a la falta de infraestructura adecuada en las escuelas para tal fin; de otro lado, a la dinámica que se traía de entregar minutas industrializadas ya preparadas (bebida achocolatada y galletas dulces). El conflicto se configuró a partir del desperdicio de alimentos dada su no utilización, por ejemplo, como ahuyama, papa, etc. Una transformación del mismo sucedió a partir de mejoras en la infraestructura de las escuelas, sensibilización de las merendeiras y capacitación para preparar menús mucho más cualificados y saludables a partir del hortifruti provisto por los agricultores familiares.

En contraste, en el caso de Granada, las manipuladoras de alimentos realizaban su labor de forma voluntaria y sin seguridad social o salario alguno. La preparación de los alimentos no se configuró como un conflicto en sí, pues, gran parte de las escuelas cuenta con una infraestructura mínima y siempre hubo una disposición por parte de las mujeres por preparar alimentos de acuerdo a las minutas establecidas. El conflicto se construye, así, por el hecho de no ser un trabajo reconocido por el operador y donde la alcaldía y en general el Estado evita establecer un vínculo laboral con las manipuladoras, constituyendo así, una forma de explotación del trabajo femenino sin remuneración alguna ${ }^{13}$. Parte de las discusiones que hoy se encuentra en el contexto colombiano es cómo crear cooperativas de manipuladoras de alimentos que a su vez puedan preparar y cualificar los alimentos, incluso, a partir de productos que ellas mismas o sus familias cultiven.

A su vez, en cada contexto existen también conflictos específicos. En el caso de São Lourenço do Sul, el tema de logística y precios, se configura como una relación de conflicto donde los actores tanto locales, como federales, disputan posiciones al

\footnotetext{
${ }^{12}$ Programa de Adquisición de Alimentos.

13 Para ampliar informaciones sobre este conflicto: https://www.desdeabajo.info/colombia/29636para-enmarcar-elmanejo-periodistico-del-fallo-de-la-corte-constitucional-a-favor-de-las-madrescomunitarias.html
} 
respecto. En este caso existe un acuerdo nacional sobre el precio que se le debe dar al agricultor familiar, éste debe ser justo, de mercado y sin intermediario.

En el caso de Granada, el conflicto más agudo se vive en el tema de trazabilidad de los alimentos y marcas. Existen al menos tres posiciones. La local, que considera que las exigencias que realiza el programa a los agricultores se fundamenta en la defensa de unos criterios de trazabilidad que solo pueden cumplir las grandes industrias y/o operadores y no los campesinos. La departamental, que se rige por el lineamiento técnico del MEN, que implica, la imposibilidad de ejercer control y vigilancia sobre los productos que suministran los campesinos. La nacional, donde un sector del Estado afirma que las exigencias que se realizan a los campesinos exceden la norma y demandan el ajuste a los lineamientos del ICBF para que abran paso a los productos locales.

En síntesis, el tema de compras públicas genera conflictos comunes a ambos territorios. Estos permiten identificar su incidencia en varios ámbitos del Estado, por lo tanto, su impacto en cuestiones de tipo estructural que demandan la construcción de políticas públicas que ataquen este tipo de problemas. En este sentido, el contexto brasilero presenta una gran riqueza en cuanto a la construcción colectiva y, con la participación activa de los actores sociales, de metodologías que ayuden a dirimir estas diferencias. En cuanto el contexto colombiano, avanza en la dirección de modificar las reglas que imposibilitan la participación de los campesinos en las compras públicas.

\section{Conclusiones}

En general, la discusión de los procesos de implementación de las políticas públicas se ha centrado en demostrar cómo el Estado logra llevar a cabo sus objetivos y metas propuestas con el fin de resolver (o no) un problema público. Por oposición, la propuesta analítica de redes de políticas públicas entiende, primero, no solo el Estado desempeña esta función; segundo, este Estado tampoco es homogéneo y está libre de intereses; tercero, no es el único actor que lidera e interviene en las políticas públicas, máxime, cuando estamos en presencia de escenarios cada vez más globalizados. En consecuencia y desde la perspectiva que aquí se abordó, las políticas públicas, como proceso político, son el resultado de la interacción entre el contexto y las relaciones sociales entre actores y agentes que intervienen en el marco de un programa de política como sucede con la alimentación escolar.

Lo anterior nos abre paso a la importancia, como bien ya lo ha destacado Klijn (1998), de valorar los contextos político-institucionales donde las redes se desenvuelven. Por consiguiente, se demostró una trayectoria convergente funcional de los programas de alimentación escolar en Colombia y Brasil, aunque, esta se da en momentos históricos diferentes. De cierta forma, la articulación alimentaciónproducción-consumo discusiones que ya fueron abordadas en el Brasil post dictadura, son precisamente las que se empiezan a desarrollar en Colombia a partir del año 2013. Si bien en el contexto brasilero estas discusiones alcanzaron un rango constitucional que difícilmente se obtendrá en el contexto colombiano, éstas si cobran un valor especial al inscribirse en el marco de la construcción de paz que, en pocas palabras, enfatiza en la seguridad alimentaria como base del desarrollo rural y 
sus políticas. Los acuerdos por esta vía adquieren ahora un valor de rango constitucional.

En clave de redes, recursos, intereses y reglas institucionales, existen tensionamientos y también formas más cooperativas. En el caso de Granada, las reglas favorecen la emergencia de conflictos interinstitucionales, principalmente, entre actores locales versus actores departamentales y nacionales. Por el contrario, en el caso de São Lourenço do Sul, las reglas formales contribuyen en crear condiciones más propicias para la agricultura familiar participar de las compras públicas. Por su parte, las reglas informales emergen como una forma de oposición en los actores locales (Granada) o como un mecanismo para desarrollar la Ley (São Lourenço do Sul). El surgimiento de estas reglas también está mediado por los intereses de los actores locales que ven en las compras públicas una alternativa viable de generación de renta para los campesinos y agricultores familiares.

Por fin, relaciones de cooperación y conflicto se entrecruzan y atraviesan varias escalas territoriales (local, regional, departamental/estadual, nacional/federal, internacional) en función de los intereses y recursos de los actores y agentes que participan del proceso y las reglas institucionales que los delimitan. Por lo tanto, las distintas configuraciones de redes en ambos países, permeadas por relaciones globales y locales específicas y diferentes trayectorias en la relación sociedad civil, Estado y mercado, interfieren en la participación de la agricultura campesina y familiar en la alimentación escolar y generan dinámicas diferenciadas en las políticas públicas.

\section{REFERENCES}

\section{ALCALDÍA DE GRANADA. Plan Municipal de Desarrollo 2016-2019. Granada Unida y} en Paz, 2016.

BOBBIO, N., Mateucci, N. e Pasquino, G. (1998). Dicionário de Política (11a ed., Vol. 1). Brasília: UNB.

BRASIL. Congresso da República. Lei 8.666. (21, junho, 1993). Regulamenta o art. 37, inciso XXI, da Constituição Federal, institui normas para licitações e contratos da Administração Pública e dá outras providências. D.O.U. Brasília, D.F., 1993. Nº 22.6.1993. pp. 1-52.

BRASIL. Ministério da Educação. Fundo Nacional de Desenvolvimento da Educação. Resolução 38. (16, julho, 2009). Dispõe sobre o atendimento da alimentação escolar aos alunos da educação básica no Programa Nacional de Alimentação Escolar PNAE. D.O.U. Brasília, D.F., 2009. Nº 17.07.2009, Seção 1. pp. 1-16.

BRASIL. Presidência da República. Casa Civil. Lei 11.947. (16, junho, 2009). Dispõe sobre o atendimento da alimentação escolar e do Programa Dinheiro Direto na Escola aos alunos da educação básica. D.O.U.. Brasília, D.F., 2009. № 17.6.2009. pp. 1-10.

BRASIL. Ministério da Educação. Fundo Nacional de Desenvolvimento da Educação. Resolução 26. (17, junho, 2013). Dispõe sobre o atendimento da alimentação escolar 
aos alunos da educação básica no âmbito do Programa Nacional de Alimentação Escolar - PNAE. D.O.U.. Brasília, D.F., 2013. N 18.06.2013. pp. 1-24

BRASIL. Ministério da Educação. Fundo Nacional de Desenvolvimento da Educação. Conselho Deliberativo. Resolução 4. (2, abril, 2015). Altera a redação dos artigos 25 a 32 da Resolução/CD/FNDE n 26, de 17 de junho de 2013, no âmbito do Programa Nacional de Alimentação Escolar (PNAE). D.O.U.. Brasília, D.F., 2015. Nº 08.04. 2015, seção 1. pp. 1-11.

BRASIL. IBGE | Brasil em Síntese | Rio Grande do Sul | São Lourenço do Sul | Panorama. Estadísticas. 2016. Disponível em: <https://cidades.ibge.gov.br/brasil/rs/sao-lourenco-do-sul/panorama>. Acesso em: 15 jan. 2018.

COLOMBIA. Congreso de la República. Ley 80. (28, octubre, 1993). Por la cual se expide el Estatuto General de Contratación de la Administración Pública. Diario oficial. Bogotá, D.C., 1993. Nº 41.094. pp. 1-28.

COLOMBIA. Ministerio de Educación Nacional. Decreto 1852. (16, septiembre, 2015). Por el cual se adiciona el Decreto 1075 de 2015 Único Reglamentario del Sector Educación, para reglamentar el parágrafo 4 o del artículo 136 de la Ley 1450 de 2011, el numeral 20 del artículo 6 de la Ley 1551 de 2012, el parágrafo 2 del artículo 2 de la Ley 715 de 2001 y los artículo 16,17,18 Y 19 de la Ley 1176 de 2007, en lo referente al Programa de Alimentación Escolar - PAE. Diario oficial. Bogotá, D.C., 2015. № 49.637. pp. 1-12.

COLOMBIA. Ministerio de Educación Nacional. Resolución 16432. (2, octubre, 2015). Por el cual se expiden los lineamientos técnico-administrativos, los estándares y las condiciones mínimas del Programa de Alimentación Escolar -PAE-. Diario oficial. Bogotá, D.C., 2015. № 49.656. pp. 1-52.

COLOMBIA. Gerencia de Seguridad Alimentaria y Nutricional de Antioquia - MANÁ - Antioquia Honesta, 2018. Disponível em: <http://www.antioquiahonesta.com/gerencias/mana/>. Acesso em: 15 jan. 2018.

COUTO DE FREIRE, Sh. Experiências na implementação do Programa Nacional de Alimentação Escolar em São Lourenço do Sul (TCC (Especialização em Gestão e Saúde)). Especialização à distância de Gestão em Saúde, UFRGS, Porto Alegre, 2012.

KLIJN, E-H. Policy Networks: An Overview. En Managing Complex Networks (pp. 245). London: Sage, 1998. Recuperado de http://revistaredes.rediris.es/webredes/textos/Complex.pdf

LÓPEZ, D. Disponibilidad de alimentos básicos en Colombia 2000-2010: ¿producción nacional o importaciones? (Disertación (Maestría en Ciencias Económicas)). Facultad de Ciencias Económicas, Universidad Nacional de Colombia, Bogotá, 2012. 
MALUF, R., SCHMITT, C. E GRISA, C. Estado de la situación del hambre y políticas de seguridad y soberanía alimentaria y de abastecimiento en los países miembros del MERCOSUR Ampliado (Relatorio técnico No. 4) (p. 69). Rio de Janeiro: Observatorio de Políticas Públicas para a Agricultura, 2009. Recuperado de http://www.observatoriodoagronegocio.com.br/page41/page42/files/CERESAN_OPP A_RelatTec4.pdf

OCARIZ, M.P. El ejercicio de la soberanía alimentaria en las familias campesinas del Valle Calchaquí - el caso de Angastaco, Salta (Disertación (Maestría en Desarrollo Rural)). Facultad de Agronomía, Universidad de Buenos Aires, Buenos Aires., 2015. Recuperado de http://ri.agro.uba.ar/files/download/tesis/maestria/20150carizmariapaula.pdf

PAULILLO, L.A. Y ALMEIDA, L.M. Gestão de redes de políticas públicas locais de segurança alimentar: uma análise comparativa dos municípios de Campinas, Araraquara e Catanduva. Gestão \& Produção, 18(4), 853-868, 2011. https://doi.org/10.1590/S0104-530X2011000400012

PORRAS, J.I. Policy Network o red de políticas públicas: Una introducción a su metodología de investigación. Estudios Sociológicos, XIX(3), 721-745, 2001. Recuperado de http://www.redalyc.org/articulo.oa?id=59805707

PROGRAMA DE LAS NACIONES UNIDAS PARA EL DESARROLLO (PNUD). Perfil productivo municipio Granada. Bogotá: PNUD-RedOrmet-Mintrabajo, 2015.

ROMERO, C. Crisis, seguridad y soberanía alimentaria en América Latina y Bolivia : de las causas y efectos a las políticas públicas, César - Autor/a; Cochabamba: IESEUMSS, 2012. Recuperado de http://biblioteca.clacso.edu.ar/Bolivia/ieseumss/20171024045822/pdf_80.pdf

RUBIO, B. Soberanía alimentaria versus dependencia: las políticas frente a la crisis alimentaria en América Latina. Mundo Siglo XXI, VII(26), 105-118, 2011. Recuperado de http://repositorio.flacsoandes.edu.ec/bitstream/10469/7116/1/REXTN-MS26-09Rubio.pdf

ZURBRIGGUEN, C. Redes, actores e instituciones. Revista del CLAD Reforma y Democracia, (30), 1-13, 2004.

Patricia Elena Giraldo Calderón. Cientista Política pela Universidade de Antioquia e Mestre em Desenvolvimento Rural pela Universidade Federal do Rio Grande do Sul (PGDR/UFRGS).pgiraldo.cal@gmail.com

Catia Grisa. Catia Grisa - doutora em Ciências Sociais (CPDA/UFRRJ). Professora no Departamento Interdisciplinar e no Programa de Pós-Graduação em 
Actores, redes y alimentos: la participación de la agricultura familiar y campesina en la alimentación escolar del Brasil y Colombia

Desenvolvimento Rural, ambos da Universidade Federal do Rio Grande do Sul. catiagrisaufrgs@gmail.com

\section{Submetido em: 18/10/2018}

\section{Aprovado em: 29/11/2018}

Como citar: CALDERÓN, Patricia Elena Giraldo; GRISA, Catia. Atores, redes e alimentos: a participação da agricultura familiar e campesina na alimentação escolar do Brasil e da Colômbia. Redes, Santa Cruz do Sul, v. 24, n. 1, p. 62-80, jan. 2019. ISSN 1982-6745. Disponível em: https://doi.org/10.17058/redes.v24i1.13049. 